\title{
NEW DEVELOPMENTS IN LIGHT SOURCE MAGNET DESIGN*
}

\author{
Soren Prestemon", Steve Marks, Ross Schlueter, LBL, Berkeley, Ca 94720, U.S.A.
}

\begin{abstract}
The rapid growth in the light source community throughout the world has served to motivate innovation in the magnet technologies that serve as the foundations for both the storage ring lattice magnet systems and the primary radiation sources, the insertion devices. Here a sampling of magnet system developments being pursued at diverse facilities are discussed, including combinedfunction magnets that minimize space requirements and improve accelerator performance, high performance bend magnets that provide enhanced radiation characteristics, and novel and untested concepts for future lattice magnets. Finally, we review developments in insertion devices that promise new performance characteristics to better serve the light source community.
\end{abstract}

\section{INTRODUCTION}

The rapid expansion in the number of light sources throughout the world has lead to a number of developments in magnet systems to address their needs. Here we will focus on a few examples of research that may play a critical role in future light sources and in improving the performance of existing sources. However, it should be noted that industry's role in reducing the cost and increasing the reliability and quality of traditional accelerator magnets for light sources has been vital in the expansion of theses sources.

Here we discuss research advances in a small sampling of magnet systems that may have a large impact on performance of light sources. These include a) combined function magnets to minimize the space requirements of the accelerator system, b) new technologies to provide unique new performance parameters and/or dramatically reduce the operating costs of the accelerator systems, and c) new developments in advanced radiation sources, the wigglers and undulators. An excellent review of insertiondevice developments was presented by P. Elleaume at EPAC [1]; we will therefore focus only on a few examples of active research. Much of the ongoing insertion device research and developments was the focus of a recent workshop hosted by the Elettra light source [2].

\section{COMBINED FUNCTION MAGNETS}

Combined function magnets have been incorporated in the lattice since the first synchrotrons were built. Recently there has been renewed interest in providing enhanced combined function performance to minimize the overall accelerator cost, improve system performance and to provide more space for the critical radiation sources, the insertion devices.
A nice example of a combined function magnet for existing light sources is the ALS sextupoles [3]. Using perturbation theory developed by Klaus Halbach [4], the traditional sextupole magnet design was modified to incorporate additional trim coils to provide independent dipole and skew-quadrupole corrections. Similar magnet systems are now commonplace on storage rings, providing correction capability without consuming vital longitudinal space (see for example [5]).

More recent examples are the systems implemented and/or being considered by MaxLab [6]. Motivated by performance, space and cost considerations, novel combined function systems are being considered with innovative pole profiles to minimize machining tolerances and costs. The proposed magnet systems for the MaxIV complex include combining sextupoles, and possibly octupoles, in the quadrupoles, with small apertures to provide strong multipole fields; concepts such as these may serve as a template for the next series of $3^{\text {rd }}$ generation light sources that will need to provide high performance while being cost-effective, with minimal performance and schedule risk.

A more unique example of combined function magnet system is the ALS permanent-magnet chicane (see Fig. 1) [7]. Using the concept of a pure-permanent magnet corrector ring [8], these systems provide independent control of a variety of multipole fields without hysteresis and with negligible power requirements. A number of devices have been in use at the ALS since 2001, and they are now the system of choice for new chicanes at the ALS. Recent design improvements include reducing the gear-ratio of the drive system to provide more accurate positioning and minimize fluctuations at the few metastable positions encountered during operation.

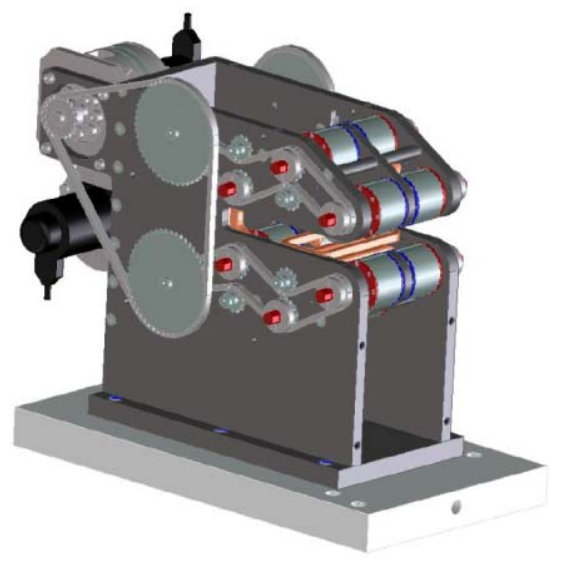

Figure 1: Model of the ALS chicane magnet, showing drive system and magnetic elements. Additional dipole correction coils are provided for higher frequency corrections. 
Note that the underlying concept of the chicane system can be used to control arbitrary multipole content and/or to serve as the principle multipole lattice element, as the strength scales with length. Similar systems are under investigation at other light sources [9], and are being considered for use in free-electron lasers [10].

\section{NOVEL TECHNOLOGIES FOR LATTICE MAGNETS}

Although the primary purpose of the lattice magnets is to provide optimal beam quality, the bend magnets are invariably used as radiation sources as well. The ALS was the first $3^{\text {rd }}$ generation light source to pursue superconducting technology for the bend magnets. In 2001 the Superbend upgrade was successfully commissioned [11]. The magnet parameters [12] were chosen to provide enhanced spectral properties for the rapidly growing field of protein crystallography. Since the lattice periodicity was fundamentally changed, the upgrade was a significant modification to the ALS both in terms of accelerator physics and in terms of spectral performance. The Superbend performance since their installation has been exemplary, with a nearly perfect operational record.

As an alternative to the ALS superconducting Superbends, the Swiss Light Source (SLS) has implemented an enhanced warm-iron dipole design to provide a $2.9 \mathrm{~T}$ field, a significant enhancement over the other 1.4T bend magnets [13]. Although not as aggressive as the Superbends, the magnets serve to illustrate the importance of maximizing bend magnet performance.

Motivated in part by the success of the ALS Superbend project, other light sources are investigating superconducting technology for the bend magnets. Technical studies of the feasibility of using hightemperature superconducting magnets for bend and multipole magnets are underway by researchers at Brookhaven [14]. The primary motivation is to minimize integrated operating costs over the system lifetime. The Brookhaven superconducting magnet group has been developing HTS react-and-wind technology for many years [15], and has demonstrated on prototype magnets that the performance requirements for such magnets are attainable. Uncertainties remain concerning the performance of such technologies in a real storage ring environment, and the long-term reliability of the systems.

The trend among $3^{\text {rd }}$ generation light sources is to migrate to full-energy injection, and in many cases TopOff mode, first implemented at the Swiss Light Source, the Advanced Photon Source, and at Spring8. Since TopOff mode does not require large field variations of the lattice magnets, non-traditional magnet systems can be considered. Permanent magnet systems can provide the field strengths needed for lattice magnets without incurring any significant operating (e.g. power and cooling) costs. The main uncertainty with the use of permanent magnets for lattice magnet application concerns radiation damage. There is evidence to suggest that radiation damage is tantamount to thermally induced demagnetization. Improved resistance to demagnetization can be provided by selecting higher coercivity material or by cooling the material to cryogenic temperatures. More directly, it may be possible to design the system geometry to reduce or eliminate radiation load. It should be noted that some correction to multipole fields will always be required to control beam quality; the ALS chicanes serve as an example for such systems. Alternatively, small electrical correction magnets could continue to be used for this purpose.

\section{DEVELOPMENTS IN RADIATION SOURCES}

Developments in insertion devices are occurring on a number of fronts. For a wide variety of users the flexibility provided by variable polarization undulator devices (EPU's) is particularly useful. Diverse permanent-magnet designs exist to provide such polarization control, although the most commonly implemented design is the APPLE II [16]. Such devices are becoming the dominant insertion device in facilities focussing on soft x-rays; example applications include magnetic linear and circular dichroism, angle-resolve photo-emission (ARPES), coherent scattering, lensless imaging, etc. [17].

For other applications, such as protein crystallography, maximum flux density at specific energies (e.g. $12.6 \mathrm{keV}$ ) is the dominant figure of merit. The motivation is then strong to reduce the period so as to access the hard $\mathrm{x}$-ray region in low undulator harmonics with maximum flux. Much effort has been focussed on improving the attainable field/period length ratio; currently the most common technology for these applications is the invacuum undulator [18]. Research is underway to improve the performance using cryogenic in-vacuum devices (CIVID) and superconducting technology (SCU).

\section{Elliptically polarizing undulator developments}

A variety of experiments benefit from control of the polarization of the radiation-field. Bend magnet radiation provides variation of the polarity by moving the experiment off the horizontal plane; planar undulators, on the other hand, provide only linear polarized radiation. To provide full polarization control, elliptically polarizing undulators were proposed [16]. The first EPU was installed on the SPEAR ring [19]; the first EPU to provide light at a $3^{\text {rd }}$ generation ring was at the ALS [20]. The most common form of the devices, the APPLE II design, are becoming the predominant insertion device in low energy rings, and are growing in popularity at high energy rings as well. Many groups now have the expertise to design, fabricate, measure and shim EPU devices. A number of industrial partners have developed the expertise as well, as the technology is now relatively mature. Issues that pose challenges in the fabrication of EPU's include: 
1. Stiff and well aligned structural backing beams and bearings between the backing beams and the magnetic carriages to minimize deformations.

2. Good quality magnetic blocks, with small variance in magnetic remanance and in the field orientation. The most commonly used magnetic materials are Somarium Cobalt $\left(\mathrm{Sm}_{2} \mathrm{Co}_{17}\right)$ and Neodymium-Iron-Boron ( $\mathrm{NdFeB})$.

3. Well-designed ends that minimize changing integrated dipole fields with changing polarization, stemming from permeability effects $[21,22]$.

Ideally insertion devices have negligible impact on the beam dynamics. Interactions can be from a) static field harmonics, such as dipole and quadrupole (normal or skew) that can be measured on the bench and compensated for via passive shimming, e.g. "virtual" shims [23] or magnetic shims [24] or from b) "dynamic" multipoles, i.e. effective multipoles emanating from field variations due to the particles trajectory. The latter do not appear in magnetic measurements, but may generate a tune-shift with undulator phase-shift that can impact the dynamic aperture. Since the particle's transverse oscillations scale with field strength $B$ and period, and the spatial field gradient is also proportional to field, dynamic effects scale with $B^{2}$ and period $\lambda$. Planar undulators provide a dynamic vertical focusing effect that can easily be accommodated by corrector magnets on the ring. Unfortunately EPU's are among the most "visible" of insertion devices due to a number of factors, including fast field roll-off intrinsic to the geometry and shiftdependent multipoles that cannot be fully compensated for using static corrections.

The recent trend among light-sources is to move towards top-off mode, where injection occurs quasicontinuously. In traditional operating regimes the insertion devices are opened during injection, both to avoid any impact from the insertion devices on injection efficiency and to avoid potential safety issues associated with unintended transmission of electrons down a beamline. In top-off mode injection occurs while experiments are proceeding, with all insertion devices operating. During injection the dynamic aperture must be significantly larger than during normal operation; the nonlinear focussing effects of the EPU devices can therefore impact injection efficiency.

A reasonable solution to this problem was developed by researchers at the ESRF [25]. Magnetic strips attached on appropriate blocks of the device generate a nonlinear horizontal kick distribution that linearizes and reduces the effective dynamic focus/defocus in most polarization modes, allowing correction from the existing corrector quadupole magnets. The technique has been demonstrated in detail at BESSY [26], and has been or will soon be implemented at many of the active 3rd generation light sources. The correction stems from magnetic material and is therefore independent of the beam; the correction therefore does not apply well to storage rings that operate in different energy modes, since the dynamic effect is proportional to $1 / E^{2}$. The effect is therefore much more pronounced on low energy (soft X-ray) machines. Other techniques are being investigated as well, including active electrical correction using line currents [27, 28].

A number of new design concepts have been proposed to generate variable polarization from superconducting magnet systems [29-31]. Analysis suggests that these designs do not provide dramatic improvements in field performance. The proposed ALS design [31] leverages the concept of period doubling / period halving to provide users access to a dramatically enlarged spectral range, without sacrificing brightness. A conceptual model of the system is shown in Fig. 2. Technical hurdles for all superconducting EPU designs include:

1. AC losses associated with field ramping typical of EPU usage

2. Superconductor hysteretic magnetization that may impact spectral quality, and

3. Beam-based heat loads that are concern for any superconducting insertion device.

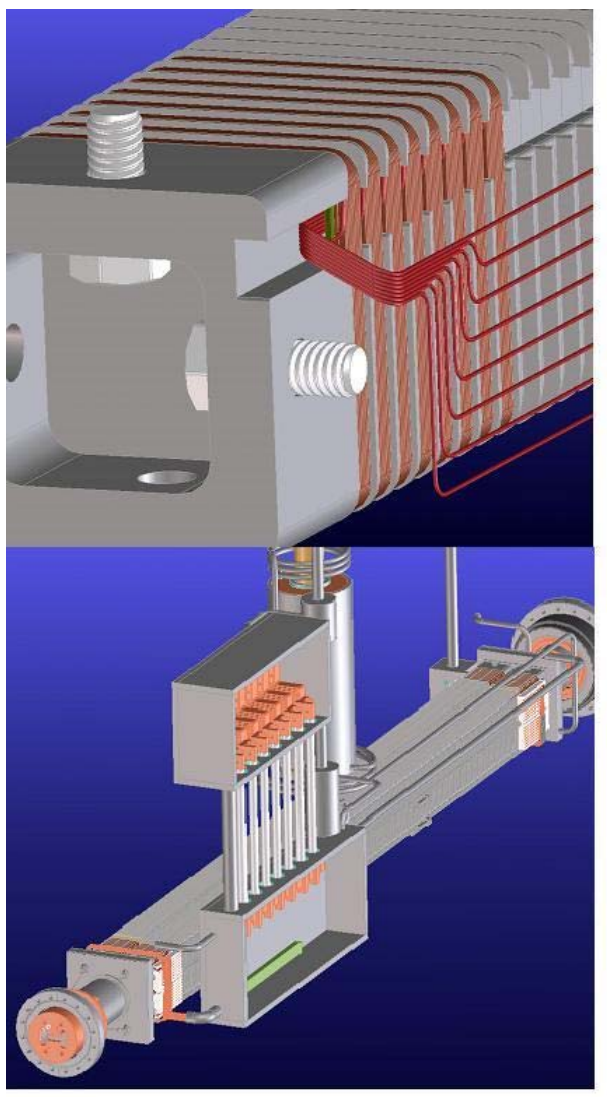

Figure 2: Superconducting elliptically polarizing undulator concept. Top: a single quadrant, with each eight coils defining the periodic structure. Bottom: the complete four-quadrant system, including a cryogenic switchyard to provide period-halving and period-doubling capabilities (see [31]).

All of these issues are addressed by the cryogenic system design and appropriate choice of conductor.

T09 Room-Temperature Magnets 


\section{Cryogenic in-vacuum undulator research}

In-vacuum undulators, first developed at Spring8, have quickly become the high-performance option for shortperiod, high brightness photon production at most operating light sources. Recently the concept of cryogenically cooling permanent magnet devices to enhance their performance was proposed by [32]. The performance improvement, estimated to be as much as $30 \%$, stems from two fronts:

1. Cooling permanent magnets to $\sim 150 \mathrm{~K}$ results in an increase in $\mathrm{Br}$ by $\sim 12 \%$, depending on the material.

2. Cooling also increases the magnetic coercivity, potentially allowing the use of higher $\mathrm{Br}$ magnetic material that cannot otherwise be used due to low room-temperature coercivity resulting in risk of demagnetization.

A number of labs have developed CIVID prototypes to determine feasibility [33]. Key areas of concern include:

- Sufficient temperature control to avoid impact on phase errors due to temperature gradients

- Shimming of the device, i.e. does room temperature shimming provide acceptable spectral properties upon cooldown

- Development of sufficiently accurate cryogenic measurement facilities

Recent data at SpRING8 suggests that room temperature phase-error correction may be sufficient for CIVID devices. Similar studies are ongoing at the ESRF; if validated, the result will eliminate a significant complication in CIVID fabrication.

Perhaps a more critical concern is that it will not be possible to bake out the device prior to installation if higher remanance material is chosen, due to thermallyinduced demagnetization of the materials stemming from the lower coercivity. If this concern is validated, the performance advantage of CIVID devices will be restricted to $\sim 12 \%$. In some cases, however, such performance may be secondary to a more useful feature: enhanced radiation hardness [34].

\section{Superconducting undulators}

The rapid development of permanent magnet insertion devices slowed the development pace for superconducting insertion devices. Permanent magnet technology has matured to the point where performance improvements are expected to be slower. The potential for superconducting devices in certain gap and period regimes is now under active research, stimulated in part by significant improvements in superconducting materials.

Since the review of superconducting insertion devices presented in [1], progress has been made on a number of issues associated with superconducting insertion devices. An SCU device fabricated by ACCEL for ANKA has been operating since 2005 [35]. Recent results of beambased heating and operational and spectral characteristics were presented in [36]. An EU funded project is underway to investigate 1) beam-based heating and appropriate cryogenic designs and 2) cryogenic measurement systems commensurate with undulator phase error determination [37].

The highest performance superconducting insertion devices use $\mathrm{Nb}_{3} \mathrm{Sn}$ superconductors, capable of 2-3 times higher current density than $\mathrm{NbTi}$ superconductors in the field range typical of short $(10-30 \mathrm{~mm})$ period undulators. Recent results at LBL have demonstrated that the technology is viable, having achieved the measured shortsample limit on a prototype device [38]. The LBL progress has stimulated other labs to investigate $\mathrm{Nb}_{3} \mathrm{Sn}$ as well, including the APS (in collaboration with the National High Magnetic Field Laboratory) [39] and ANKA [37].

Outstanding issues with superconducting undulators include

- Thermal loads, in particular quantification of beam-induced heat loads in the case of cold-bore operation; a transportable calorimeter designed to be installable on a variety of storage rings would provide critical information on these loads and their ring parameter dependence, and could be funded, designed, and built via a light source collaboration

- Shimming for phase error correction of higher harmonics. Although preliminary concepts of active shimming have been tested [40, 41], research is needed to develop a complete system compatible with user operation.

The research and development of these devices has progressed to make them viable candidates for a variety of light source applications in the near future. Applications of CIVID and SCU devices on a variety of rings will provide a useful set of data to determine longterm viability of the technologies.

\section{REFERENCES}

[1] J. Chavanne, P. Elleaume, Proceedings EPAC 2006.

[2] B. Diviacco, "New Frontiers in Insertion Devices", ELETTRA Users Meeting Satellite Workshop, Nov. 20-21, 2006.

[3] S. Marks, "Magnetic Design of Trim Excitations for the Advanced Light Source Storage Ring Sextupole", IEEE Transaction on Magnetics, Vol. 32, No. 4. Proceedings of 14th Magnet Technology Conference, Tampere, Finland, June 11-16, 1995.

[4] K. Halbach, "First Order Perturbation Effects in IronDominated Two-Dimensional Symmetrical Multipoles", Nuclear Instruments and Methods, Vol $74,1969$.

[5] A. Nadji, J. Neel, P. Peaupardin, "Analysis of the Multipurpose Sextupole of Soleil Using Halbach's Perturbation Theory and the Opera-2D Code", Proceedings of EPAC 1996.

[6] H. Tarawneha, M. Erikssona, L-J. Lindgrena, B. Anderberg, "MAX-IV lattice, dynamic properties and magnet system", Nuclear Instruments and Methods in 
Physics Research A, 508 (2003); also M. Sjostrom, H. Tarawneh, E. Wallen, M. Eriksson, Characterization of the Max II Storage Ring Lattice", Nuclear Instruments and Methods in Physics Research A, May 2007.

[7] S. Marks, R. Schlueter, D. Anderson, W. Gath, J. Y. Jung, D. Robin, C. Steier, T. Stevens, "New Chicane Magnet Design for Insertion Device Straights at the Advanced Light Source", LBNL-49292.

[8] R. Schlueter, D. Humphries, J. Tanabe, "Pure Permanent Magnet Harmonics Corrector Ring", Nuclear Instruments and Methods in Physics Research A, 395 (1997).

[9] J. Bahrdt, Private Communication

[10] J. Pfluger, "Planar Permanent Magnet Quadrupoles for the X-FEL: A Proposal", presentation Workshop on Undulator systems for X-FEL's (WUS2005), June 6-8, 2005, DESY Hamburg, Germany.

[11]C. Steier, T. Byrne, L. Nadolski, D. Robin, B. Salvant, T. Scarvie, Commissioning of the ALS with Superbends", Proceedings of EPAC 2002, Paris, France.

[12] J. Zbasnik, et al., "ALS Superbend Magnet System", IEEE Transactions on Applied Superconductivity, vol. 11, No. 1, pp 2531-2534, March 2001.

[13] A. Ludeke, A. Andersson, M. Boge, B. Kalantari, B. Keil, M. Pedrozzi, T. Schilcher, A. Streun, V. Schlott, "status of the Swiss Light Source", Proc. EPAC 2006

[14] J. Murphy, NSLS Accelerator Division Report, 2006.

[15] R. Gupta et al., "R\&D for Accelerator Magnets with React and Wind High Temperature Superconductors", IEEE Transactions on Applied Superconductivity, Vol. 12, No. 1, March 2002.

[16]S. Sasaki, Nuclear Instruments and Methods in Physics Research A 347, 83 (1994).

[17] J. Kirz, D. S. Chemla, B. Feinberg, Z. Hussain, G.F. Krebs, H. A. Padmore, D. S. Robin, A. L. Robinson, N. V. Smith, T. Warwick, "Advanced Light Source Strategic Plan", March 2006. Wwwals.lbl.gov/als/ourorg/strategicplan.pdf

[18] T. Hara, T. Tanaka, T. Tanabe, X. M. Marechal, S. Okada, H. Kitamura, J. Synch. Rad 5 (1998) 403

[19]R. Carr, J. B. Kortright, M. Rice, S. Lidia, "Performance of the Elliptically Polarizing Undulator on SPEAR", SLAC-PUB-95-6627.

[20]S. Marks et al., "The Advanced Light Source Elliptically Polarizing Undulator", Proceedings PAC 1997, Vancouver.

[21] J. Chavanne, P. Elleaume, P. Van Vaerenbergh, "End Field Structure For Linear/Helical Insertion Devices", Proceedings of the 1999 Particle Accelerator Conference, New York, 1999

[22]R. Schlueter, S. Marks, S. Prestemon, "Elliptically Polarizing Undulator End Designs", IEEE Transactions on Applied Superconductivity, Vol 16., No. 2, June 2006

[23] S. Marks, J. DeVries, E. Hoyer, B.M. Kincaid, D. Plate, P. Pipersky, R.D. Schlueter, A. Young,
Proceedings of the 1999 Part. Acc. Conference, New York, NY, USA, 1999, pp. 162-164.

[24] D.C. Quimby, S.C. Gottschalk, F.E. James, K.E. Robinson, J.M. Slater and A.S. Valla. Nucl. Instr. and Meth. A 285 (1989), p. 281.

[25] J. Chavanne et al., "Recent Achievements and Future Prospects of ID Activities at the ESRF", Proceedings EPAC 2000.

[26]P. Kuske, "Effects of Fringe Fields and Insertion Devices Revealed Through Experimental Frequency Map Analysis", Proceedings of PAC 2005.

[27] Ingvar Blomquist, ADC Workshop, Feb. 18, 2005

[28] J. Safranek, Personal Communication.

[29] B. Kostka, R. Rossmanith, Proceedings of APAC 2004.

[30] S. H. Kim, C. Doose, Proceedings of PAC 2005.

[31] S. Prestemon, R. Schlueter, S. Marks, D. Dietderich, MT19.

[32] T. Hara, T. Tanaka, H. Kitamura, T. Bizen, X. Marechal, T. Seike, PRST-AB 7 (2004).

[33] These include SpRING8, ESRF, NSLS; see presentation of T. Tanaka, J. Chavanne, G. Rakowsky, respectively at "New Frontiers in Insertion Devices", ELETTRA Users Meeting Satellite Workshop, Nov. 20-21, 2006. The NSLS effort is in collaboration with industrial partner ADC (see presentation by E. Johnson, same Workshop).

[34] G. Rakowski, Private Communication

[35]B. Kosta, R. Rossmanith, D. Wollmann, M. Hagelstein, Proceedings of the 2005 PAC Conference, p. 2559 (2005).

[36] S. Casalbuoni, M. Hagelstein, B. Kostka, R. Rossmanith, M. Weisser, E. Steffens, A. Bernhard, D. Wollmann, T. Baumbach, "Performance of the first superconducting cold-bore undulator in an electron storage ring", Presented at ASC 2006.

[37]R. Rossmanith, "New Frontiers in Insertion Devices", ELETTRA Users Meeting Satellite Workshop, Nov. 20-21, 2006.

[38] D.R. Dietderich, H.C. Higley, A. Godeke, S.O. Prestemon, P.T. Pipersky, R.D. Schlueter, S. Marks, "Fabrication of a Short-Period Nb3Sn Superconducting Undulator", Presented at ASC 2006.

[39] H.W. Weijers, K.R. Cantrell, L. Marks, J.R. Miller, "Assembly procedures for a Nb3Sn undulator demonstration magnet", Presented at ASC 2006.

[40] S. O. Prestemon, et al., "Design, Fabrication and Test Results of Undulators Using Nb3Sn Superconductor", IEEE Trans. on Appl. Superconf., June 2005 (presented at ASC 2004, Jacksonville, Fl.).

[41] C. S. Hwang, J. C. Jan, P. H. Lin, C. H. Chang, M. H. Huang, F. Y. Lin, T. C. Fan, and H. H. Chen, "Minipole Superconducting Undulator for X-Ray Synchrotron Light Source", IEEE Transaction on Applied Superconductivity, Vol 16, No 2., June 2006. 